\title{
Analgesic Efficacy of TAP Block Versus Wound Subfascial Infiltration After Inguinal Hernia Surgery: Randomized Prospective Study
}

Mounir Khalil ${ }^{*}$, Lamkinsi Tarik ${ }^{2}$, Bibich Lotfi ${ }^{3}$, Jbili Nabil ${ }^{3}$, Diai Abdellatif ${ }^{3}$, Chkoura Khalid ${ }^{3}$, Laoutid Jaouad ${ }^{3}$, Kechna Hicham ${ }^{3}$, Hachimi Moulay Ahmed ${ }^{3}$

\author{
${ }^{1}$ Mohammed V Military Hospital, Rabat, Morocco \\ ${ }^{2}$ Laboratoire de Génétique et Biométrie, Université IBN Tofail - Kenitra, Avenida de L'Université, Kénitra, Morocco \\ ${ }^{3}$ Military Hospital Moulay Ismail, Meknès, Morocco
}

DOI: $10.36347 /$ sasjm.2022.v08i01.005

| Received: 05.12.2021 | Accepted: 08.01.2022 | Published: 20.01.2022

*Corresponding author: Khalil Mounir

Mohammed V Military Hospital, Rabat, Morocco

\section{Abstract}

Original Research Article

Introduction: The main objective of this study is to compare TAP block with subfascial wound infiltration in the prevention of acute and chronic postoperative pain after surgical repair of inguinal hernia. Materials and methods: This is a prospective randomized study. After approval by the local ethics committee and obtaining informed patients consent, ASA I or ASA II patients proposed for a simple inguinal hernia cure are included. Patients were randomized into two groups: Subfascial cicatricial infiltration with $0.5 \%$ bupivacaine $(20 \mathrm{ml})$ during wall closure (Group 1); a TAP block (by the technique of two projections) with $0.5 \%$ bupivacaine $(20 \mathrm{ml})$ on the operated side (Group 2). Postoperative analgesia is provided by systematic Paracetamol and Nefopam and morphine titration. Apart from demographic parameters and ASA class, the postoperative pain intensity at rest and at coughing, the morphine consumption and the secondary effects were compared. Patients' satisfaction and postoperative chronic pain at 3 and 6 months were also analyzed. Results: Concerning demographic parameters, ASA class and secondary effects, we didn't find any meaningful difference. However, there was a significant reduction of postoperative pain in the TAP group whether at rest as coughing. Gr 1 patients asked for more morphine consumption and they were less satisfied and accused more chronic pain. Conclusion: This study shows that the TAP block is as simple and effective technique in reducing acute postoperative pain and preventing chronic pain after inguinal hernia surgery. This technique seems well tolerated and more effective than a single subfascial infiltration injection.

Keywords: Inguinal hernia; infiltration; TAP block; postoperative pain; chronic pain.

Copyright $\odot 2022$ The Author(s): This is an open-access article distributed under the terms of the Creative Commons Attribution 4.0 International License (CC BY-NC 4.0) which permits unrestricted use, distribution, and reproduction in any medium for non-commercial use provided the original author and source are credited.

\section{INTRODUCTION}

Most patients suffer from moderate to severe pain after inguinal hernia repair [1]. Despite being considered as simple and minor, this surgery is responsible of both acute and chronic pain [2]. If acute pain affects the early recovery period, chronic pain can seriously affect the quality of life of patients in the long term. Therefore, the concern for postoperative analgesia should not be limited to the immediate postoperative period but also the prevention of chronic pain. Improvement of postoperative pain represents a particular interest to patients, surgeons, and anesthetists. As part of multimodal analgesia, multiple methods have been put into use to provide postoperative comfort, such as local anesthetic infiltration, epidural analgesia, peripheral nerve block, and patient-controlled analgesia [3]. Wound infiltration is still a simple and effective technique in postoperative pain reduction [4]. The transversus abdominis plane (TAP) block may provide analgesia to the parietal peritoneum as well as the skin and muscles of the anterior abdominal wall [5].

The main objective of this study is to compare the efficacy and efficiency of TAP block with subfascial wound infiltration in the prevention of acute and chronic postoperative pain after surgical repair of inguinal hernia.

\section{PATIENTS AND METHODS}

This prospective randomized comparative clinical trial was conducted in anesthesia service of Moulay Ismail Military Hospital of Meknes in Morocco. This work lasted from Jun 2017 to February 2018. After local ethical committee approval and 
patients' informed written consents, 80 patients of ASA physical status I and II scheduled for open unilateral inguinal hernia repair under spinal anesthesia were included. All patients who did not give their consent and/or with a history of allergy to local anesthetics, neurological or psychiatric history, anticoagulant therapy or antiplatelet therapy in addition to acetylsalicylic acid or a history of renal or hepatic failure were excluded from the study. In order to avoid confusion bias, all patients were operated by the same surgical team and the same operative technique. During the pre-anesthetic visit, patients were allocated randomly using sealed envelopes, according to a computer-generated sequence of random numbers, into two equal groups:

- Group 1 or infiltration received wound infiltration with $20 \mathrm{ml}$ of isobaric bupivacaïne $0.5 \%$ (AGUETTANT) during the parietal closure.

- Group 2 or TAP received non ultrasound-guided TAP block with $20 \mathrm{ml}$ of isobaric bupivacaïne $0.5 \%$ (AGUETTANT) on the same side as the hernia after parietal closure.

After randomization, we performed a posteriori verification of the comparativity of the 2 groups.

The anesthetic protocol was standard for all patients. Premedication was performed with $2 \mathrm{mg} / \mathrm{kg}$ of hydroxyzine orally administered the day before surgery. Under a standard monitoring system (non-invasive pressure, electrocardioscope and pulsed oxygen saturation), a peripheral venous pathway allowed intake of $250 \mathrm{ml}$ of $0.9 \%$ saline serum. An Oxygen flow rate of $2 \mathrm{l} / \mathrm{min}$ was administered to all patients. After surgical asepsis in sitting position, the L3-L4 or L4-L5 interspinous space was punctured by $25 \mathrm{G}$ Whitacre needle allowing spinal administration of $12.5 \mathrm{mg}$ of hyperbaric bupivacaine. Targeted anesthetic level was T11.

For all patients, Lichtenstein technique was performed for inguinal hernia repair by the same surgery team. A propylene mesh was placed to fit the floor of the inguinal canal and its apex was sutured to the pubic tubercle using a 3-0 prolene suture. After making an opening to accommodate the spermatic cord, the lower border of the synthetic prosthesis was attached to the free edge of the inguinal ligament. Finally, the mesh was anchored to the conjoint tendon by interrupted sutures.

In the infiltration group, at the end of the procedure, and before closing the surgical wound, the anesthesiologist gave the surgeon a $20-\mathrm{ml}$ syringe filled completely with the infiltration fluid (bupivacaine $0.5 \%$ ). The surgeon infiltrated the subcutaneous tissue after making sure the injection was extravascular.
In the TAP block group, while the separation field of operation is still in place, rigorous asepsis was performed and the Jean-Louis and Petit triangle in operated side was identified. The puncture, without ultrasound guidance, was performed using anesthetic needle purged with $0.5 \%$ bupivacaine filled in a $20 \mathrm{ml}$ syringe. After passage of the cutaneous plane, the identification of the two projections and a negative aspiration test, slow and split injection was realized. The TAP block was done by the same practitioner for all patients.

As a single blind approach, no patient knew from the beginning which analgesic technique will be performed.

Postoperative analgesia was performed by an infusion of Paracetamol 1g/8h and Nefopam $20 \mathrm{mg} / 8 \mathrm{~h}$. Intravenous morphine titration $(1 \mathrm{mg} / 1 \mathrm{mg})$ was performed when necessary.

Postoperative pain intensity at rest and in coughing efforts using visual analogue pain score (VAS) was evaluated at hour H0, H1, H2, H4, H8, H12, H24, H36 and H48.

A part of demographic parameters and ASA class, the postoperative pain intensity at rest and at coughing (VAS), and the morphine consumption within 48 hours were compared. Secondary effects, and patient's satisfaction were evaluated at discharge time. Three satisfaction levels were proposed: satisfied, unsatisfied and indifferent.

Postoperative chronic pain at 3 and 6 months were also analyzed. Four levels were proposed; absent, mild, moderate and severe.

Absent pain was defined by the total absence of pain or discomfort at the site of surgery with a normal return to the previous lifestyle. Mild pain was defined by the presence of occasional pain or discomfort with no limitation of activity. Moderate pain was defined by the inability to resume sport activity or to lift objects as before surgery. Severe pain was defined as continuous or intermittent pain but forbidding daily activity such as walking with the need for analgesic medication.

The primary endpoint chosen was the reduction of at least $25 \%$ of the pain scores in the TAP group compared to the Infiltration group. Having chosen a second beta species risk of $80 \%$, the minimum number computed for our study was 74 patients divided into 2 groups. We opted to add $10 \%$ for more security. This led to 80 patients in total.

All observations were entered and coded on Windows Excel 2010 (Microsoft, USA) and analyzed 
using SPSS Statistics 24 software (IBM, Chicago, IL, USA).

Excel software was also used to present tables and graphs, as well as to group the terms of some variables before their analysis.

For qualitative variables, comparison of percentages required the Pearson chi-square test with Yates correction when necessary for small samples (less than 5 observations).

Quantitative variables are expressed in means +/- standard derivations when the distribution is normal. In this case, the comparison is made using the parametric Student $t$ test for two independent groups or paired as required.

In the opposite case, the qualitative variables are expressed in median and interquartiles [Q1 - Q3]. In order to compare the two groups concerning pain, we used the Wilcoxon test because of the pairing between the patients.

In order to perform the statistical comparison, we have chosen as the threshold of significance, a risk of the first species $\alpha$ equal to 0.05 .

\section{RESULTS}

A total of eighty patients were included in the study (forty patients in each group). Patients demographic parameters, ASA class and surgical duration, were comparable between two groups (Table 1). VAS at rest was significantly lower in the TAP as compared to the infiltration group at all the time points except immediately postoperatively in the operating room and at first postoperative hour (Fig 1). On coughing, VAS was significantly lower in the TAP group at all the time points except in $\mathrm{H} 0$ and $\mathrm{H} 1$ (Fig 2). Concerning the morphine consumption, Gr 1 patients needed an average dose significantly higher than that of Gr 2 patients $(0.95 \mathrm{mg}$ versus $0.25 \mathrm{mg} ; p<0.009)$ (Fig $3)$.

No signs of cardiovascular or neurological toxicity of bupivacaine was noted in our patients. A part of one case of pain on injection site in TAP group, it was the same concerning the complications linked to the infiltration or TAP block gesture (hematoma of the wall, wall infection...). In addition, some minor morphine side effects were noted but without significant difference between the two groups (Table 3 ).

Concerning satisfaction status, most satisfied patients were in TAP group (82.5\% versus 62.5\%; $p<0.001)$. There was no difference between groups in indifferent patients (Table 2).

Three months after the intervention, $92.5 \%$ of Gr 2 patients had no pain, while $35 \%$ of Gr 1 patients had moderate pain. The difference in this pain was in favor of Gr 2 with statistical significance for absent pain and moderate pain. Six months after surgery, $67.5 \%$ of TAP group had patients no pain and $42.5 \%$ of infiltration group patients accused moderate chronic pain. No patient reported a severe pain in two groups patients (Fig 4).

Table 1: Demographic parameters, ASA status and surgery duration of surgery

\begin{tabular}{|l|l|l|l|l|}
\hline & TOTAL & INFILTRATION & TAP & $p$-value \\
\hline Sex ratio (M/F) & $61 / 19(76 / 24 \%)$ & $31 / 9(77 / 3 \%)$ & $30 / 10(75 / 25 \%)$ & NS \\
\hline Age (years) & $47(9,2)$ & $46.5(8.6)$ & $47.5(9.8)$ & NS \\
\hline Height $(\mathrm{cm})$ & $172(0.7)$ & $173(0.05)$ & $170(0.8)$ & NS \\
\hline Weight $(\mathrm{kg})$ & $76.2(8)$ & $75.7(9.4)$ & $76.7(6.6)$ & $\mathrm{NS}$ \\
\hline ASA Status n (\%) : I/II & $67 / 11(86 / 14 \%)$ & $33 / 5(87 / 13 \%)$ & $34 / 6(85 / 15 \%)$ & $\mathrm{NS}$ \\
\hline Duration of the surgical procedure (min) & $48.9(14.9)$ & $47.3(14.8)$ & $48.8(15.2)$ & NS \\
\hline
\end{tabular}

Demographics and ASA status are expressed as main (SD) or number (percentage); $\mathrm{p}$-value refers to the comparison between the two sub-groups for the a posteriori check of the randomization 


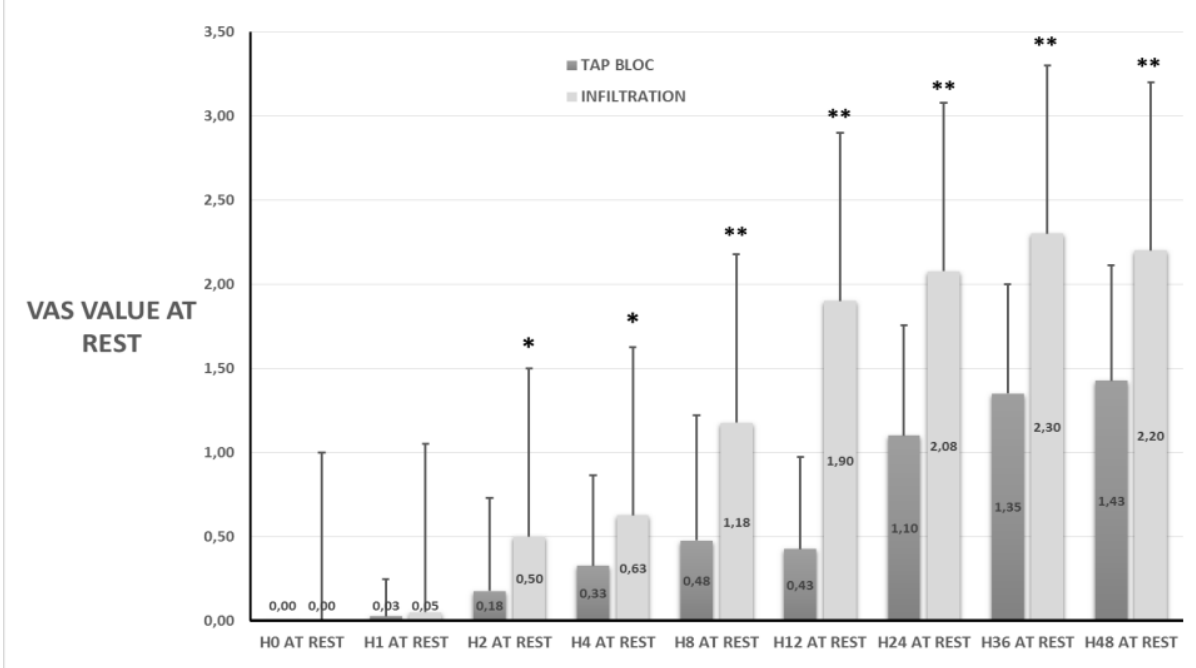

Figure 1: Pain score (VAS) at rest; *: $p<0,05$ and $* *: p<0,001$

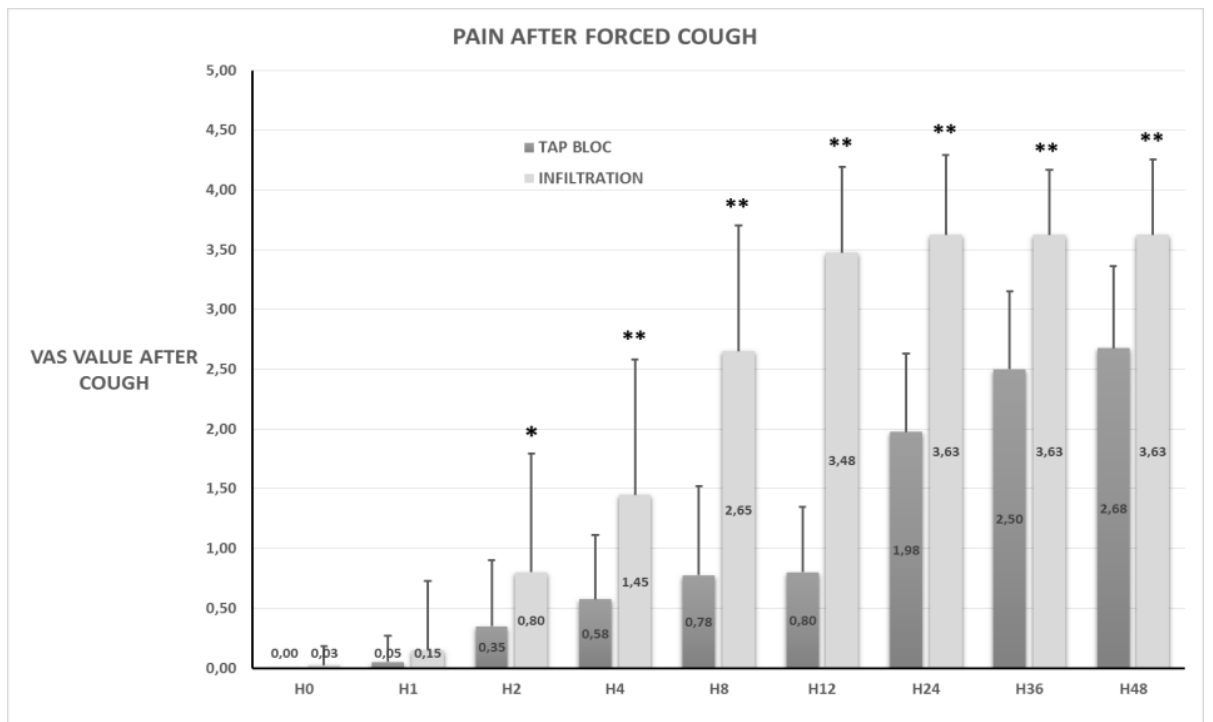

Figure 2: Pain score (VAS) at coughing; *: p<0,05 and **: p<0,001

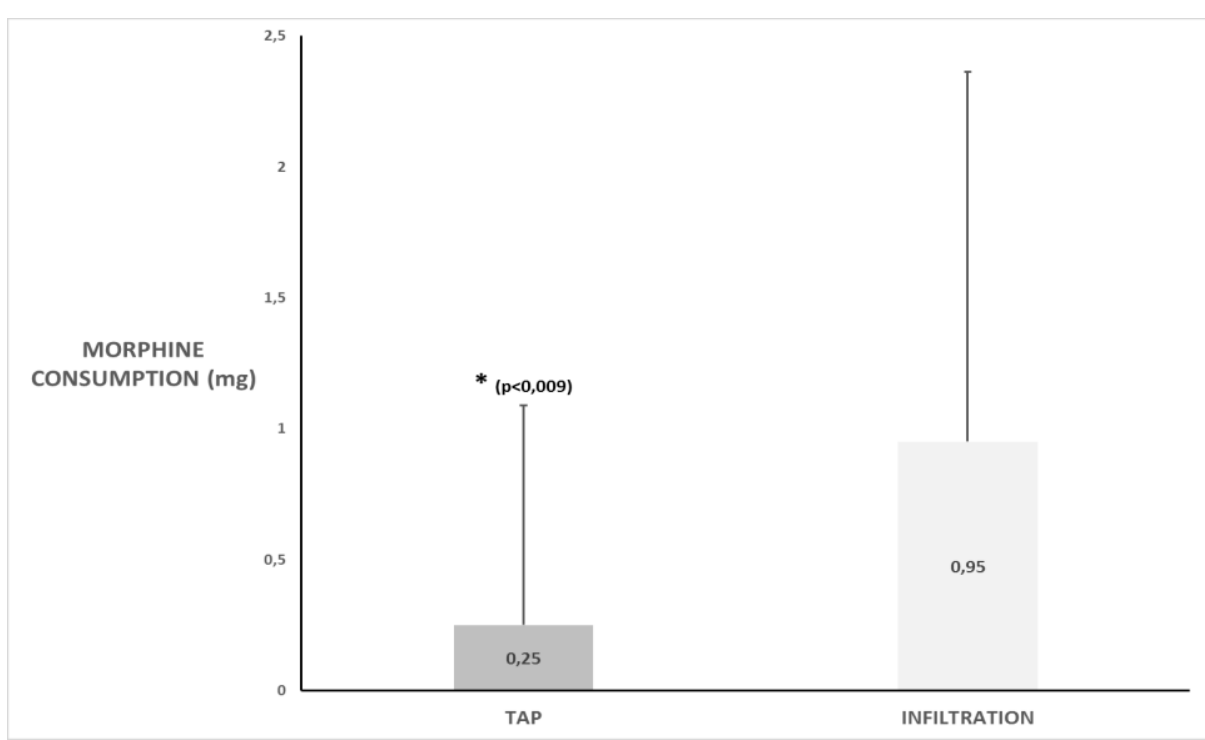

Figure 3: Morphine consumption (mg) 


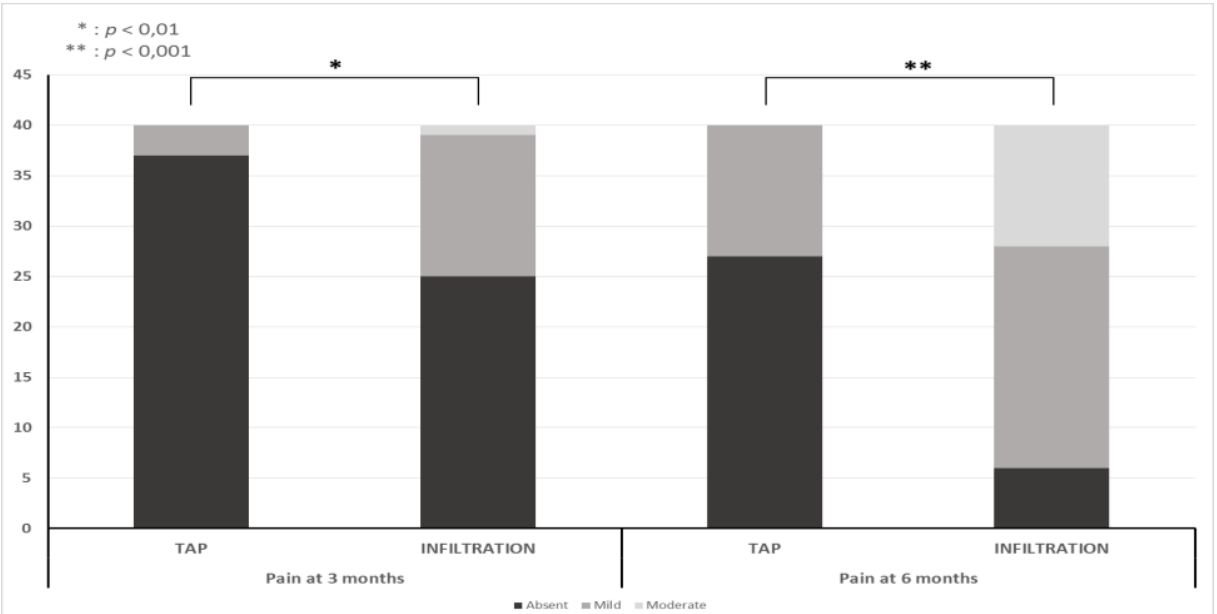

Figure 4: Chronic pain three and six months after surgery (absent, mild and moderate)

Table 2: Comparison of satisfaction status; results are expressed in number (percentage)

\begin{tabular}{|l|l|l|l|}
\hline & Groupe 1 & Groupe 2 & $\mathrm{p}$ \\
\hline Satisfied n (\%) & $25(62,5)$ & $33(82,5)$ & $<0,001$ \\
\hline Dissatisfied n (\%) & $5(12,5)$ & $1(2,5)$ & $<0,001$ \\
\hline Indifferent n (\%) & $10(25)$ & $6(15)$ & NS \\
\hline
\end{tabular}

Table 3: Postoperative side effects; results are expressed as number (percentage)

\begin{tabular}{|l|l|l|l|}
\hline & Groupe 1 & Groupe 2 & $p$ \\
\hline Nausea n (\%) & $1(2,5)$ & $2(5)$ & NS \\
\hline Vomiting n (\%) & $1(2,5)$ & 0 & NS \\
\hline Pain in site injection n (\%) & 0 & $1(2,5)$ & NS \\
\hline
\end{tabular}

\section{DISCUSSION}

This randomized, single blind, controlled trial has demonstrated that TAP block as a simple technique, is effective in reducing acute postoperative pain and preventing chronic pain after hernia repair. This technic seems well tolerated and more effective than a single injection subfascial infiltration. Acute postoperative pain is a common problem after lower abdominal surgery [3], like a hernia repair. This pain causes prolonged hospital stays and patient dissatisfaction [6]. As a result, all the analgesic techniques find an interest in it. Moreover, opioids don't have to be shown to be associated with dose-related side effects. In fact, nausea, vomiting, and gastrointestinal paralysis may be detrimental to a patient's recovery after surgery [7]. As component of multimodal analgesic approach, both wound infiltration and TAP block seem to be efficient. $\mathrm{Yu}$ et al found in their meta-analysis that both local anesthetic infiltration and TAP block are comparable in short term analgesic effects with a small advantage for TAP block which has better long-lasting effect [3]. Since the 90's, the wound infiltration by local anesthesia has been described as a simple and effective [8, 9]. In the same decade, Kuppuvelumani et al., realize the first description of TAP block [10], and eight years later Rafi publishes the first detailed documentation of this bloc [11]. Innervating the abdominal wall, the lower 6 intercostal afferent nerves represent the target of TAP bloc [12]. Several techniques are currently used to realize the block, such as blinded double POP technique [11], ultrasoundguided [13] or laparoscopic-assisted approaches [14-16]. Ultrasound-guided TAP block, which allows more accurate visualization of the needle, TAP plane, and injection spot, is considered to be safe clinically and in the same sense of ideas, the laparoscopic approach would be safer. In our study, the blinded double POP technique was chosen for two reasons; first, the impossibility of ensuring the availability of the ultrasound machine in the operating room when we wanted to perform the TAP block; second, it was considered that the use of the ultrasonograph will give an advantage to the block TAP compared to infiltration. The comparison between these two techniques has been the subject of numerous studies and meta analysis. Abd El Hamid et al., in their patients operated for hernia repair under general anesthesia, conclude that TAP block provided more reliable and effective analgesia and less of total 24 hours postoperative morphine consumption compared with wound infiltration [17].

Despite of some limits, such as the significant heterogeneity of the outcomes and mostly the small sample size of patients included studies (only four randomized controlled trials), metaanalysis published by $\mathrm{Yu}$ et al found that TAP block is comparable to infiltration for short-term analgesia and may also provide better long-lasting analgesia especially at 24 hour after lower surgery [3]. Including 791 patients from 8 heterogeneous randomized controlled trials, a metaanalysis performed by Gao et al conclude that TAP 
block reduces postoperative morphine requirements and the severity of pain after hernia surgery [18]. The realization of TAP block is enameled of the risk of numerous complications. In fact, block failure, vascular injury, abdominal viscera and nerve injuries are the common complications [19]. No complication was reported in this study as well as several randomized controlled trials [20-23]. Bupivacaine was the local anesthetic used in our study and its toxic dose in a single administration is $175 \mathrm{mg}$ [24], which allows a safety when $100 \mathrm{mg}$ are used.

Chronic pain is the most common late complication of inguinal hernia surgery [25]. To talk about chronic pain, the necessary range is variable in the literature according to the studies [26-31]. Moreover, chronic pain is defined as persistent pain beyond 3 months [32]. In our study, it was evaluated at three and six postoperative months. The same surgical technique was performed by the same surgeon team for all patients. Available treatments have an often partial and modest effectiveness. Therefore, chronic pain may be considered as a programmed pathology that is better to prevent [33]. Two main mechanisms are responsible for chronic pain; direct or indirect nerves injuries [34, 35] and neuroplasticity [36]. Thus, wound infiltration and TAP block can both theoretically prevent this pain. A preventive effect of the subfascial infiltration was previously found [2]. Furthermore recently, a randomized controlled trial comparing ultrasoundguided TAP bloc with $20 \mathrm{ml}$ of ropivacaine $0.75 \%$ to placebo conclude that the incidence of chronic pain was low and not significantly affected by the performance of the block.

The satisfaction of the majority of Gr 2 patients shows the effectiveness and safety of the TAP block and demonstrate that this block can be used as a component of analgesic postoperative strategy.

To our knowledge, there is no trial in literature which compare analgesic effect of TAP block to wound infiltration during 48 postoperative hours or even more their preventive potential for chronic pain.

\section{Limits of the study}

Even though our study still original trial, it remains true that there are certain limits such as small sample size, monocentric study and the use of morphine titration instead of patient controlled analgesia.

\section{Declarations of interest: none}

\section{REFERENCES}

1. Rawal, N., Hylander, J., Nydahl, R. A., Olofsson, I., \& Gupta, A. (1997). Survey of postoperative analgesia following ambulatory surgery. Acta Anaesthesiologica Scandinavica, 41(8), 1017-1022.

2. Mounir, K., Bensghir, M., Elmoqaddem, A., Massou, S., Belyamani, L., Atmani, M., \&
Azendour, H. (2010, February). Efficiency of bupivacaine wound subfasciale infiltration in reduction of postoperative pain after inguinal hernia surgery. In Annales francaises d'anesthesie et de reanimation, 29(4), pp. 274-278.

3. Yu, N., Long, X., Lujan-Hernandez, J. R., Succar, J., Xin, X., \& Wang, X. (2014). Transversus abdominis-plane block versus local anesthetic wound infiltration in lower abdominal surgery: a systematic review and meta-analysis of randomized controlled trials. BMC anesthesiology, 14(1), 1-9.

4. Beaussier, M., \& Aissou, M. (2009, March). Single-shot wound infiltration for postoperative analgesia. Neurosurgery, ENT, thoracic abdominal and perineal surgery. In Annales francaises d'anesthesie et de reanimation, 28(3), pp. e163e173.

5. Young, M. J., Gorlin, A. W., Modest, V. E., \& Quraishi, S. A. (2012). Clinical implications of the transversus abdominis plane block in adults. Anesthesiology Research and Practice, 2012, 731645.

6. Mhuircheartaigh, R. N., Moore, R. A., \& McQuay, H. J. (2009). Analysis of individual patient data from clinical trials: epidural morphine for postoperative pain. British Journal of Anaesthesia, 103(6), 874-881.

7. Jørgensen, H., Wetterslev, J., Møiniche, S., \& Dahl, J. B. (2001). Epidural local anaesthetics versus opioid-based analgesic regimens for postoperative gastrointestinal paralysis, PONV and pain after abdominal surgery. Cochrane database of systematic reviews, (1).

8. Dahl, J. B., Møiniche, S., \& Kehlet, H. (1994). Wound infiltration with local anaesthetics for postoperative pain relief. Acta anaesthesiologica scandinavica, 38(1), 7-14.

9. Renck, H. (1994). Wound infiltration with local anaesthetics. Acta anaesthesiologica scandinavica, 38(1), 2-6.

10. Kuppuvelumani, P., Jaradi, H., \& Delilkan, A. (1993). Abdominal nerve blockade for postoperative analgesia after caesarean section. Asia-Oceania Journal of Obstetrics and Gynaecology, 19(2), 165-169.

11. Rafi, A. N. (2001). Abdominal field block: a new approach via the lumbar triangle. Anaesthesia, 56(10), 1024-1026.

12. Tran, T. M. N., Ivanusic, J. J., Hebbard, P., \& Barrington, M. J. (2009). Determination of spread of injectate after ultrasound-guided transversus abdominis plane block: a cadaveric study. British journal of anaesthesia, 102(1), 123-127.

13. Hebbard, P., Fujiwara, Y., Shibata, Y., \& Royse, C. (2007). Ultrasound-guided transversus abdominis plane (TAP) block. Anaesthesia and intensive care, 35(4), 616-618.

14. Chetwood, A., Agrawal, S., Hrouda, D., \& Doyle, P. (2011). Laparoscopic assisted transversus abdominis plane block: a novel insertion technique 
during

nephrectomy. Anaesthesia, 66(4), 317-318.

15. Elamin, G., Waters, P. S., Hamid, H., O'Keeffe, H. M., Waldron, R. M., Duggan, M., ... \& Khan, I. Z. (2015). Efficacy of a laparoscopically delivered transversus abdominis plane block technique during elective laparoscopic cholecystectomy: a prospective, double-blind randomized trial. Journal of the American College of Surgeons, 221(2), 335344.

16. Keller, D. S., Ermlich, B. O., Schiltz, N., Champagne, B. J., Reynolds Jr, H. L., Stein, S. L., $\&$ Delaney, C. P. (2014). The effect of transversus abdominis plane blocks on postoperative pain in laparoscopic colorectal surgery: a prospective, randomized, double-blind trial. Diseases of the Colon \& Rectum, 57(11), 1290-1297.

17. Abd El-Hamid, A. M., \& Afifi, E. E. (2016). Transversus abdominis plane block versus local anesthetic wound infiltration in patients undergoing open inguinal hernia repair surgery. Ain-Shams Journal of Anaesthesiology, 9(2), 280-283.

18. Gao, T., Zhang, J. J., Xi, F. C., Shi, J. L., Lu, Y., Tan, S. J., \& Yu, W. K. (2017). Evaluation of Transversus Abdominis Plane (TAP) block in hernia surgery. The Clinical journal of pain, 33(4), 369-375.

19. Soltani Mohammadi, S., Dabir, A., \& Shoeibi, G. (2014). Efficacy of transversus abdominis plane block for acute postoperative pain relief in kidney recipients: a double-blinded clinical trial. Pain Medicine, 15(3), 460-464.

20. Petersen, P. L., Mathiesen, O., Stjernholm, P., Kristiansen, V. B., Torup, H., Hansen, E. G., ... \& Dahl, J. B. (2013). The effect of transversus abdominis plane block or local anaesthetic infiltration in inguinal hernia repair: a randomised clinical trial. European Journal of Anaesthesiology (EJA), 30(7), 415-421.

21. Atim, A., Bilgin, F., Kilickaya, O., Purtuloglu, T., Alanbay, I., Orhan, M. E., \& Kurt, E. (2011). The efficacy of ultrasound-guided transversus abdominis plane block in patients undergoing hysterectomy. Anaesthesia and intensive care, 39(4), 630-634.

22. Sivapurapu, V., Vasudevan, A., Gupta, S., \& Badhe, A. S. (2013). Comparison of analgesic efficacy of transversus abdominis plane block with direct infiltration of local anesthetic into surgical incision in lower abdominal gynecological surgeries. Journal of anaesthesiology, clinical pharmacology, 29(1), 71-75.

23. Skjelsager, A., Ruhnau, B., Kistorp, T. K., Kridina, I., Hvarness, H., Mathiesen, O., \& Dahl, J. B. (2013). Transversus abdominis plane block or subcutaneous wound infiltration after open radical prostatectomy: a randomized study. Acta Anaesthesiologica Scandinavica, 57(4), 502-508.
24. Boogaerts, J. (1998). Pharmacologie des anesthésiques locaux. In: Fondation européenne d'enseignement en anesthésiologie (FEEA). Edition Pradel; p. 281-312.

25. Keller, J. E., Stefanidis, D., Dolce, C. J., Iannitti, D. A., Kercher, K. W., \& Heniford, B. T. (2008). Combined open and laparoscopic approach to chronic pain after inguinal hernia repair. The American Surgeon, 74(8), 695-701.

26. Poobalan, A. S., Bruce, J., King, P. M., Chambers, W. A., Krukowski, Z. H., \& Smith, W. C. S. (2001). Chronic pain and quality of life following open inguinal hernia repair. Journal of British Surgery, 88(8), 1122-1126.

27. MRC Laparoscopic Groin Hernia Trial Group. (1999). Laparoscopic versus open repair of groin hernia: a randomised comparison. The Lancet, 354(9174), 185-190.

28. McGillicuddy, J. E. (1998). Prospective randomized comparison of the Shouldice and Lichtenstein hernia repair procedures. Archives of surgery, 133(9), 974-978.

29. Callesen, T., Bech, K., Thorup, J., Andersen, J., Nielsen, R., Roikjaer, O., \& Kehlet, H. (1998). Cryoanalgesia: effect on postherniorrhaphy pain. Anesthesia \& Analgesia, 87(4), 896-899.

30. Juul, P., \& Christensen, K. (1999). Randomized clinical trial of laparoscopic versus open inguinal hernia repair. Journal of British Surgery, 86(3), 316-319.

31. Aasvang, E., \& Kehlet, H. (2005). Chronic postoperative pain: the case of inguinal herniorrhaphy. British journal of anaesthesia, 95(1), 69-76.

32. Martinez, V., Ammar, S. B., Judet, T., Bouhassira, D., Chauvin, M., \& Fletcher, D. (2012). Risk factors predictive of chronic postsurgical neuropathic pain: the value of the iliac crest bone harvest model. Pain, 153(7), 1478-1483.

33. Eisenach, J. C. (2006). Preventing chronic pain after surgery: who, how, and when?. Regional anesthesia and pain medicine, 31(1), 1-3.

34. Eisenberg, E. (2004). Post-surgical neuralgia. Pain, 111(1), 3-7.

35. Aasvang, E. K., Brandsborg, B., Christensen, B., Jensen, T. S., \& Kehlet, H. (2008). Neurophysiological characterization of postherniotomy pain. PAIN®, 137(1), 173-181.

36. Simonnet, G. (2008). Preemptive antihyperalgesia to improve preemptive analgesia. The Journal of the American Society of Anesthesiologists, 108(3), 352-354.

37. Theodoraki, K., Papacharalampous, P., Tsaroucha, A., Vezakis, A., \& Argyra, E. (2019). The effect of transversus abdominis plane block on acute and chronic pain after inguinal hernia repair. A randomized controlled trial. International Journal of Surgery, 63, 63-70. 